\title{
BMJ Open Protocol for a systematic review of health promotion interventions for African Americans delivered in US barbershops and hair salons
}

\author{
Kelly Palmer (D) , ${ }^{1}$ Patrick Rivers, ${ }^{1}$ Forest Melton, ${ }^{1}$ Jean McClelland ${ }^{2}$ \\ Jennifer Hatcher, ${ }^{3}$ David G Marrero, ${ }^{1}$ Cynthia Thomson, ${ }^{1}$ David O Garcia ${ }^{1}$
}

To cite: Palmer K, Rivers $P$, Melton F, et al. Protocol for a systematic review of health promotion interventions for African Americans delivered in US barbershops and hair salons. BMJ Open 2020;10:e035940. doi:10.1136/ bmjopen-2019-035940

- Prepublication history and additional material for this paper are available online. To view these files, please visit the journal online (http://dx.doi. org/10.1136/bmjopen-2019035940).

Received 22 November 2019 Revised 26 March 2020 Accepted 07 April 2020

Check for updates

(C) Author(s) (or their employer(s)) 2020. Re-use permitted under CC BY-NC. No commercial re-use. See rights and permissions. Published by BMJ.

${ }^{1}$ Department of Health Promotion Sciences, College of Public Health, University of Arizona, Tucson, Arizona, USA

${ }^{2}$ Health Sciences Library, University of Arizona Arizona Health Sciences Center, Tucson, Arizona, USA

${ }^{3}$ Division of Public Health Practice and Translational Research, University of Arizona, Phoenix, Arizona, USA

Correspondence to

Kelly Palmer;

kpalmer1@email.arizona.edu

\section{ABSTRACT}

Introduction African American adults are

disproportionately burdened by chronic diseases, particularly at younger ages. Developing culturally appropriate interventions is paramount to closing the gap in these health inequities. The purpose of this systematic review is to critically evaluate health promotion interventions for African Americans delivered in two environments that are frequented by this population: barbershops and hair salons. Characteristics of effective interventions will be identified and evidence for the effectiveness of these interventions will be provided. Results of this review will inform future health promotion efforts for African Americans particularly focused on the leading health inequities in obesity-related chronic diseases: cardiovascular disease, cancer and type 2 diabetes.

Methods and analysis Subject headings and keywords will be used to search for synonyms of 'barbershops,' 'hair salons' and 'African Americans' to identify all relevant articles (from inception onwards) in the following databases: Academic Search Ultimate, Cumulative Index of Nursing and Allied Health Literature, Embase, PsycINFO, PubMed, Web of Science (Science Citation Index and Social Sciences Citation Index) and ProQuest Dissertations. Experimental and quasi-experimental studies for adult $(\geq 18$ years) African Americans delivered in barbershops and hair salons will be included. Eligible interventions will include risk reduction/management of obesity-related chronic disease: cardiovascular disease, cancer and type 2 diabetes. Two reviewers will independently screen, select and extract data and a third will mediate disagreements. The methodological quality (or risk of bias) of individual studies will be appraised using the Effective Public Health Practice Project Quality Assessment Tool. Quality and content of the evidence will be narratively synthesised. Ethics and dissemination Since this is a protocol for a systematic review, ethical approval is not required. Findings from the review will be widely disseminated through conference presentations, peer-reviewed publications and traditional and social media outlets.

\section{INTRODUCTION}

African Americans constitute 13.4\% of the US population making them the second
Strengths and limitations of this study

- This is the first systematic review to assess health promotion interventions targeted for African Americans delivered in barbershops and hair salons.

- Six major databases and one grey literature database will be searched to obtain all eligible studies.

- There may be a limited number of eligible studies.

- Heterogeneity of study outcomes may hinder ability to compare the effectiveness of intervention strategies.

largest minority group. ${ }^{1}$ While life expectancy rates for all US adults have increased from 1997 to 2015, the mortality rates for African Americans are still disproportionately higher than non-Hispanic whites (NHW). ${ }^{2}$ Moreover, African Americans are experiencing health inequities related to obesity and related chronic conditions such as cardiovascular disease, cancer and type 2 diabetes at younger ages than NHW driving up morbidity and mortality rates in young to middle adulthood. ${ }^{2}$ African Americans have the highest prevalence $(49.6 \%)$ of obesity and severe obesity of any racial/ethnic group. ${ }^{3}$ They are twice as likely to have a stroke or die from heart disease, and $50 \%$ more likely to have high blood pressure than NHW. ${ }^{4}$ Cancer disparities are astounding for African Americans. African Americans have a higher incidence of colorectal cancer and are twice as likely to die from multiple myeloma and stomach cancer than NHW. ${ }^{5}$ African American men are more than twice as likely to die from prostate cancer, while African American women are more likely to die from breast and cervical cancer than $\mathrm{NHW}^{5}$ At $12.7 \%$, African Americans have the second highest prevalence of diagnosed type 2 diabetes. ${ }^{6}$ African Americans, a disadvantaged and marginalised group, are vulnerable to human 
rights violations and health inequities. Social determinants such as wage inequality, unfair housing laws and reduced access to quality education and healthcare influence health outcomes by creating barriers or pathways to risk factors. ${ }^{78}$ African Americans are also less likely to see a primary care doctor on a regular basis due to costs, lack of trust in the healthcare system and other sociocultural factors. ${ }^{89}$ Similarly, African Americans are under-represented in health promotion research. ${ }^{10-12}$ There is an urgent need to develop effective, culturally appropriate health promotion interventions to engage African Americans to mitigate conditions that lead to health inequities.

Community partnerships and trusted sources of healthcare, such as same race providers, community health workers and medical organisations that focus on African Americans, can play an integral role in reducing cultural barriers to care, supporting coordinated care or linking people to resources, delivering health promotion interventions, and identifying socioeconomic and other factors that contribute to health disparities. Many health promotion interventions have struggled to engage African Americans. This is likely due to a lack of cultural adaption of curricula since most interventions are not designed with cultural factors in mind. Interventions are oftentimes placed in environments that are not viewed as easily accessible or are associated with racial prejudice and therefore do not meet people where they work, live and play. To remedy the issues with health promotion engagement and to tackle health inequities among African Americans, researchers and health programme interventionists have looked to community-based interventions that are more culturally appropriate and acceptable.

Faith-based organisations have served as a prime venue for health behaviour interventions among African Americans. $^{13} 14$ Historically among African Americans, the church has been a place not only for spiritual worship, but as a safe haven to socialise and engage in civic and political action. The church provides personal and economic development and community outreach. The African American church offers social support/structure and religiosity, core cultural constructs among African Americans that when incorporated into interventions yield positive outcomes. ${ }^{15}$ Many faith-based interventions incorporate scripture and prayer into curricula to provide a deeper connection and reason for adopting healthy behaviours. ${ }^{15}$ Involving church leadership in delivering the intervention or supporting/reinforcing engagement in the intervention use role modelling and the social network that is important to African Americans. ${ }^{16}$ Conversely, faith-based and faith-placed interventions have limitations. African American men and young adults are less likely to attend church regularly than African American women and older adults. ${ }^{17}$ Black churches have been inundated with various initiatives making it difficult to engage the church in implementing health promotion interventions. ${ }^{18}$

An alternative environment to capitalise on the sociocultural factors that influence African Americans' behaviour are barbershops and hair salons. Barbershops and hair salons, much like the church, have been staples of the African American community. These highly accessible establishments have been sources of entrepreneurship for African Americans earning respect for not only the owners, but for the barbers and stylists as well. Barbershops and hair salons provide important African American cultural constructs such as communalism and expressiveness. ${ }^{19}$ Hair salons and barber shops have been used in different aspects of health promotion research such as cancer screening, hypertension, and fruit and vegetable consumption involving formative research to recruitment of study participants to sites for the delivery of interventions. ${ }^{19-26}$ Barbershop-based and barbershopplaced interventions have become increasingly popular over the last decade. ${ }^{27}$ Barbershops have been a valuable resource for reaching African American men, a traditionally difficult group to engage in research and the healthcare sector. Many African American men spend hours at the barbershop even when not being serviced. This time is spent networking for jobs, storytelling, purchasing various goods from African American vendors, enjoying entertainment (movies, sports, etc) and participating in other recreation (board games, dominoes, etc). ${ }^{28} 29$ The majority of research that has taken place in barbershops address hypertension (screening and treatment adherence) and prostate cancer screening. ${ }^{27}$ Interventions have been evaluated based on delivery by the researcher versus by the barber. ${ }^{27}$ Because of systematic racism, mistrust of the medical system, reduced access to healthcare and sociocultural attitudes, it is not uncommon for an African American woman to have an established source of hair care, but to not have a primary care provider. ${ }^{30}$ This speaks to the importance hair and hair care plays in the lives of African American women. African American women will undergo hair care that can be time consuming, financially burdensome, physically uncomfortable and unhealthy (due to exposure to chemicals, etc.) with regularity. ${ }^{31}$ African American women spend considerable amount of time and money at hair salons thereby creating a captured audience suitable for health behaviour interventions. ${ }^{31}$ Research around hair and hair care behaviours among African American women as it relates to health behaviour have centred on hair as a barrier to physical activity. ${ }^{32}$ Studies using hair stylists to deliver health communication around breast cancer screening and other health behaviours have shown promise due to the unique and close relationship African American women have with their stylist. ${ }^{33-38}$

\section{Rationale}

Very few systematic reviews have looked at site or setting for engaging African Americans in health promotion, primarily focusing on 'cultural adaptions' of evidencebased interventions. ${ }^{39-43}$ Of those that have, they were largely focused on recruitment strategies of participants for clinical trials from sites that include churches, barbershops and hair salons. ${ }^{445}$ One synthesis of the literature 
examined interventions delivered in barbershops and hair salons but did not focus on African Americans as the target population..$^{20}$ In 2015 , a qualitative systematic review was conducted describing health promotion interventions for African American men delivered by barbers, but did not include interventions delivered in hair salons or that focused on African American women. ${ }^{27}$ To date, there have been no published systematic reviews of interventions delivered in hair salons targeting African American women. This will be the first systematic review of health promotion interventions that combines both African American men and women delivered in either barbershops and hair/beauty salons.

\section{Objective}

The aim of this systematic review is to critically examine existing health promotion interventions for African Americans delivered in barbershops and hair salons and to understand if there is quality evidence for the effectiveness of these interventions. By identifying characteristics of effective interventions, this will be useful to inform future efforts for health promotion for African Americans, with emphasis on the leading health inequities in chronic diseases in this population: heart disease, cancer, stroke and type 2 diabetes.

\section{METHODS AND ANALYSIS}

\section{Study registration}

This systematic review protocol has been submitted for registration with the International Prospective Register of Systematic Reviews (PROSPERO) and is pending acceptance; date of submission: 18 November 2019. The protocol adheres to the reporting guidelines of the Preferred Reporting Items for Systematic Reviews and Meta-Analysis Protocol (PRISMA-P) statement. ${ }^{46}$ The PRISMA-P checklist is provided as online supplementary file 1 . Since this study is a systematic review of published literature, ethical approval will not be required. Significant protocol amendments will be documented and published with the results of the review.

\section{Eligibility criteria}

Study inclusion will be restricted to studies published in English. The types of study designs, participants, interventions, comparators and outcomes that will be considered for inclusion are discussed below and outlined in table 1 .

\section{Study designs}

We will include experimental and quasi-experimental study designs including randomised control trials, nonequivalent post-test only, and pre-post-test studies. Descriptive studies, observational studies (cross-sectional studies, case-control, cohort studies, case reports and

Table 1 Inclusion and exclusion criteria

\begin{tabular}{ll}
\hline PICOS strategy & Inclusion criteria \\
\hline P-Population & African American/black, male and female, \\
& $18+$ years old, living in the USA
\end{tabular}

\section{Exclusion criteria}

Studies that recruited both African Americans/ blacks and non-African Americans where the focus was not on African Americans/blacks (separate analysis for African Americans not conducted), studies focused on children and/or adolescents ( $<18$ years of age).

I-Intervention Interventions delivered in barbershops or hair salons/beauty shops with a health-related (obesity/chronic condition) outcome

$\begin{array}{ll}\text { C-Comparison } & \text { For RCTs, wait-list control, attention } \\ & \text { control, usual care or standard treatment. } \\ & \text { For quasi-experimental, no comparison } \\ & \text { required. } \\ & \text { Studies reporting outcomes related to } \\ \text { h-Outcome } & \text { heart disease, cancer screening and type } \\ & 2 \text { diabetes as a primary or secondary } \\ & \text { outcome. }\end{array}$

S-Study design

RCTs, factorial, non-equivalent post-test only, and pretest studies and post-test study designs. biochemical and biomarker testing for environmental exposures, interventions delivered by massage therapists, interventions delivered in nail and tanning salons and interventions delivered outside of the USA.

Study outcomes related to dermatological diseases (tinea, alopecia, reactions to chemical hair treatments), side effects of cancer treatment and environmental exposures.

Reviews, observational studies (cross sectional case-control and cohort studies), case reports, case series, animal studies, secondary analyses of trials, qualitative studies and survey development studies.
Studies involving the collection of hair for 
case series), animal studies, secondary analyses of trials and survey development studies will not be included.

\section{Participants}

Studies will be included in this review that recruited adult (18+years) African Americans only. African American adults living in the USA are disproportionately affected by heart disease, cancer, stroke and type 2 diabetes. ${ }^{4}$ The aforementioned diseases are the leading causes of death for non-communicable diseases among adult African Americans. ${ }^{4}$ Traditionally, African Americans in the USA have been difficult to engage in health promotion efforts and research due to a longstanding mistrust of the medical and research community. ${ }^{9}$ Careful consideration must be given to designing and implementing interventions that speak to cultural nuances specific to this community.

\section{Interventions}

Studies that take place in sites that can be characterised as barbershops or hair/beauty salons that cater to an African American clientele will be included and examined for feasibility, efficacy and effectiveness in delivering health promotion interventions aimed at reducing risk factors or to improve health outcomes of obesity and related chronic conditions (ie, cardiovascular disease, cancer and type 2 diabetes). This will include weight loss/maintenance, dietary, physical activity, screening and treatment adherence interventions.

\section{Comparators}

The intervention groups may be compared with: no active treatment, usual care, attention controls, waitlist controls, alternative treatments, active treatment in a different setting or with a non-African American population.

\section{Study outcomes}

For inclusion in this review, studies must report an outcome, participant-reported or objective assessment, related to heart disease/stroke (blood pressure, medication/treatment adherence and lipids), cancer (screening) or type 2 diabetes (screening, glucose, lipids and medication/treatment adherence) as the primary or secondary outcome of interest. This will also include changes in weight and/or BMI, diet and physical activity.

\section{Information sources}

A comprehensive search will be conducted by JM for all relevant articles using the search terms detailed in online supplementary file 2 from inception to October 2019 in the following databases: Academic Search Ultimate, Cumulative Index of Nursing and Allied Health Literature, Embase, PsycINFO, PubMed and Web of Science. We will also search the ProQuest Dissertation database. To ensure comprehensiveness, reference lists from all eligible studies will be hand-searched to identify additional studies for inclusion.

\section{Search strategy}

Two thematic areas of focus related to population (African Americans and blacks) and intervention sites (barbershops, barber, hair salon and beauty culture) guided the following Medical Subject Headings (MeSH) terms and keywords used in the search: "African American," "Black American," "African Ancestry," "barber," "barbering," "beautician," "beauty culture," "cosmetologist," "hair," "hairdresser," "hairstylist," "stylist," "beauty shop,' "beauty salon," "hair salon,' and "salon."

\section{Study records}

\section{Data management}

Studies identified through the search of the electronic databases will be exported by JM into EndNote V.X9 citation manager software and duplicate records will be removed. The EndNote file will then be imported to Covidence systematic review software (Veritas Health Innovation, Melbourne, Australia) and re-checked for duplicates. KP, PR, and FM will then independently begin the reviewing process.

\section{Selection process}

After excluding duplicates, articles generated from the search strategy will be divided into two equal sections (A and $\mathrm{B}$ ). The three authors (KP, PR and FM) will independently perform an initial title and abstract review in the following manner: KP will review all articles A and B, PR will review articles A and FM will review articles B. FM will handle discrepancies for articles A and PR will handle discrepancies for articles B. In the next step, remaining articles will be read in full text format independently by KP and PR. Discrepancies at these two screening steps will be resolved by DOG and the set of articles to be reviewed will be finalised.

\section{Data extraction}

Data from these articles will be extracted independently by KP and PR and reviewed by FM. To ensure consistency and standardisation of data extraction, a Research Electronic Data Capture (REDCap) database will be developed specifically for this review. REDCap is a secure, web-based application designed to support data capture and storage for research. ${ }^{47}$ Data reports will be cross-referenced by DOG for any discrepancies. If data extraction for an eligible study cannot be accomplished due to information inadequately described or missing in the full text article, KP will contact the publication's corresponding author via email up to three times to request the information. KP and PR will pilot the REDCap database on a subsample of eligible studies and make any appropriate adjustments to the data collection fields as necessary before continuing with the remaining studies. In the case of an intervention having multiple publications meeting eligibility criteria, only the manuscript presenting the final data assessment point will be included. Intervention materials, such as educational materials provided to participants, will be 
requested from trial authors and published as supplementary materials in the final review. ${ }^{48}$

Data to be extracted from eligible articles will include:

1. Author(s) and year of publication.

2. Participant characteristics (sample size, age, sex, socioeconomic status, important eligibility criteria such disease status or risk factors, geographic location).

3. Disease state/focus (cardiovascular disease, cancer, type 2 diabetes, obesity).

4. Study design (randomised-controlled trial or quasiexperimental design).

5. Study setting (barbershop, hair salon, both).

6. Intervention variables (duration and follow-up time points, CBPR approach (yes, no), interventionist (research team, barber/stylist, other), culturally-sensitive strategies implemented, supplemental materials and theoretical frameworks/behaviour change techniques).

7. Comparator (if available) and description.

8. Study outcome data (screening completion, medication/treatment adherence, weight loss and/or changes in BMI, and changes in risk factors for chronic disease (eg, diet, physical activity, blood glucose, haemoglobin A1c and lipids) ) included as primary or secondary outcomes and feasibility outcomes of acceptability (satisfaction and intention to continue), practicality, integration and limited efficacy.

9. For interventions delivered by barbers/stylists: recruitment strategies, intervention training and measures of fidelity.

\section{Outcomes and prioritisation}

Outcomes of interest for this study include healthrelated results as defined by each study from the interventions as well as measures of feasibility and fidelity. Studies with significant findings will be reported in the final review. Efficacy will be difficult to assess due to the various uncontrolled variables (site, interventionist, sample characteristics, etc) among these 'real world' studies. Effectiveness will be assessed by improvements in the reduction of risk factors such as blood pressure, glucose and weight and by changes in health behaviours related to risk such as changes in diet, physical activity and screening adherence. We will also note limitations of each study such as threats to validity, sample size and bias, and generalisability. These findings will identify factors that impact intervention effectiveness and how they can be refined.

\section{Quality assessment}

Quality in all studies will be independently assessed by KP and PR using the Effective Public Health Practice Project Quality Assessment Tool (EPHPP). ${ }^{49}$ This standardised tool, developed by the Effective Public Health Practice Project in Canada, was chosen for its high interrater reliability and lower risk of bias. ${ }^{50}$ Also, it can be used to evaluate a range of intervention study designs. The EPHPP evaluates the study quality by assessing the following six domains: (1) selection bias; (2) study design; (3) confounders; (4) blinding; (5) data collection and (6) withdrawals/dropouts. When using this tool, each of these six domains are rated from weak (one point) to strong (three points) and these are averaged to provide a total quality score for the study. Validity and reliability for this tool meets accepted standards. ${ }^{41950}$ For the current review, all studies to be included in the review will have a copy of the EPHPP attached, and both KP and PR will independently assess each article. Any discrepancies will be resolved by DOG. Since we are not conducting a metaanalysis, there will be no data synthesis, and therefore no sensitivity analysis will be conducted with the results from this assessment.

\section{DATA ANALYSIS}

To give a comprehensively broad overview synthesising the effectiveness of health promotion interventions for African Americans delivered in barbershops and hair salons, we will present findings narratively in text and tables. It is anticipated that studies in this review will vary widely and be extremely heterogeneous; therefore, this textual approach is appropriate. We expect that the literature on this topic will be scarce and, therefore, we do not want to further limit potential studies for inclusion in order to pool data for a meta-analysis. In recognition of this limitation, for study outcomes that are sufficiently homogeneous, we will include quantitative summaries of extracted data. KP will synthesise all the data and this will be reviewed by PR. Discrepancies will be resolved by DOG. These summaries will be used to assess the effectiveness of interventions delivered in barbershops and hair/beauty salons for African Americans in the USA and address gaps in the existing literature. After synthesising the data, we will identify areas for future research.

\section{PATIENT AND PUBLIC INVOLVEMENT}

As this systematic review will be based on previously published data, there will be no patient and/or public involvement in the design, interpretation or dissemination of the findings.

\section{ETHICS AND DISSEMINATION}

Since this is a protocol for a systematic review, ethical approval is not required. Any amendments made to this protocol while conducting the study will be reported in PROSPERO and in the final published manuscript. Findings from the review will be widely disseminated through conference presentations, peer-reviewed publications, and traditional and social media outlets.

Acknowledgements We would like to thank our colleagues for their continuous support.

Contributors KP designed and drafted the review protocol, registered the review, will coordinate the review process, and is the guarantor of the systematic review. 
$\mathrm{KP}, \mathrm{JM}, \mathrm{JH}, \mathrm{DGM}, \mathrm{CT}$, and DOG contributed to the review's initial conception and revised the manuscript. KP, JM, and DOG developed the search strategies and performed the search. KP, PR, FM, and DOG will contribute to identifying studies to review, data collection and analysis. JH, DGM, CT, and DOG revised and edited the protocol manuscript.

Funding The authors have not declared a specific grant for this research from any funding agency in the public, commercial or not-for-profit sectors.

Competing interests None declared.

Patient and public involvement Patients and/or the public were not involved in the design, or conduct, or reporting, or dissemination plans of this research.

Patient consent for publication Not required.

Provenance and peer review Not commissioned; externally peer reviewed.

Open access This is an open access article distributed in accordance with the Creative Commons Attribution Non Commercial (CC BY-NC 4.0) license, which permits others to distribute, remix, adapt, build upon this work non-commercially, and license their derivative works on different terms, provided the original work is properly cited, appropriate credit is given, any changes made indicated, and the use is non-commercial. See: http://creativecommons.org/licenses/by-nc/4.0/.

\section{ORCID iD}

Kelly Palmer http://orcid.org/0000-0002-4818-6030

\section{REFERENCES}

1 United States Census Bureau. QuickFacts- Population estimatesRace and Hispanic Origin [Table], 2018. Available: https://www. census.gov/quickfacts/fact/table/US/PST045218

2 Cunningham TJ, Croft JB, Liu Y, et al. Vital Signs: Racial Disparities in Age-Specific Mortality Among Blacks or African Americans - United States, 1999-2015. MMWR Morb Mortal Wkly Rep 2017;66:444-56.

3 Hales CM, Carroll MD, Fryar CD, et al. Prevalence of obesity and severe obesity among adults: United States, 2017-2018. Hyattsville, MD: National Center for Health Statistics, 2020.

4 Blackwell D, Lucas JW, Clarke T. Summary health statistics for U.S. adults: National health interview survey, 2012. National Center for Health Statistics, 2014.

5 Ward EM, Sherman RL, Henley SJ, et al. Annual report to the nation on the status of cancer, featuring cancer in men and women age 2049 years. J Natl Cancer Inst 2019;111:1279-97.

6 Centers for Disease Control and Prevention. National diabetes statistics report, 2017; 2017.

7 Braveman P, Gottlieb L. The social determinants of health: it's time to consider the causes of the causes. Public Health Rep 2014;129 (Suppl 2):19-31.

8 Noonan AS, Velasco-Mondragon HE, Wagner FA. Improving the health of African Americans in the USA: an overdue opportunity for social justice. Public Health Rev 2016;37:12.

9 Boulware LE, Cooper LA, Ratner LE, et al. Race and trust in the health care system. Public Health Rep 2003;118:358-65.

10 Branson RD, Davis K, Butler KL. African Americans' participation in clinical research: importance, barriers, and solutions. Am J Surg 2007;193:32-9.

11 Brown Speights JS, Nowakowski ACH, De Leon J, et al. Engaging African American women in research: an approach to eliminate health disparities in the African American community. Fam Pract 2017;34:322-9.

12 Buchholz SW, Wilbur J, Schoeny ME, et al. Retention of African American women in a lifestyle physical activity program. West $J$ Nurs Res 2016;38:369-85.

13 Whitt-Glover MC, Borden SL, Alexander DS, et al. Recruiting African American churches to participate in research: the learning and developing individual exercise skills for a better life study. Health Promot Pract 2016;17:297-306.

14 Lancaster KJ, Carter-Edwards L, Grilo S, et al. Obesity interventions in African American faith-based organizations: a systematic review. Obes Rev 2014;15 (Suppl 4):159-76.

$15 \mathrm{Kim} \mathrm{KH}$-cheon, Linnan L, Campbell MK, et al. The word (wholeness, oneness, righteousness, deliverance): a faith-based weight-loss program utilizing a community-based participatory research approach. Health Educ Behav 2008;35:634-50.

16 Kumanyika S, Taylor WC, Grier SA, et al. Community energy balance: a framework for contextualizing cultural influences on high risk of obesity in ethnic minority populations. Prev Med 2012;55:371-81.
17 Grant CG, Davis JL, Rivers BM, et al. The men's health forum: an initiative to address health disparities in the community. J Community Health 2012;37:773-80.

18 Markens S, Fox SA, Taub B, et al. Role of black churches in health promotion programs: lessons from the Los Angeles mammography promotion in churches program. Am J Public Health 2002:92:805-10.

19 Linnan L, Thomas S, D'Angelo H. African American barbershops and beauty salons. Community organizing and community building for health and welfare. 229, 2012.

20 Linnan LA, D'Angelo $\mathrm{H}$, Harrington CB. A literature synthesis of health promotion research in salons and barbershops. Am J Prev Med 2014;47:77-85.

21 Johnson LT, Ralston PA, Jones E. Beauty salon health intervention increases fruit and vegetable consumption in African-American women. J Am Diet Assoc 2010;110:941-5.

22 Hess PL, Reingold JS, Jones J, et al. Barbershops as hypertension detection, referral, and follow-up centers for black men. Hypertension 2007;49:1040-6.

23 Davis C, Darby K, Moore M, et al. Breast care screening for underserved African American women: community-based participatory approach. J Psychosoc Oncol 2017;35:90-105.

24 Floyd TD, DuHamel KN, Rao J, et al. Acceptability of a Salon-Based intervention to promote colonoscopy screening among African American women. Health Educ Behav 2017;44:791-804.

25 Forte DA. Community-based breast cancer intervention program for older African American women in beauty salons. Public Health Rep 1995;110:179-83.

26 Luque JS, Roy S, Tarasenko YN, et al. Feasibility study of engaging Barbershops for prostate cancer education in rural African-American communities. J Cancer Educ 2015;30:623-8.

27 Luque JS, Ross L, Gwede CK. Qualitative systematic review of barber-administered health education, promotion, screening and outreach programs in African-American communities. J Community Health 2014;39:181-90.

28 Wright E II, Calhoun TC. From the common thug to the local businessman: an exploration into an urban African American barbershop. Deviant Behavior 2001;22:267-88.

29 Fading ABK. Twisting, and Weaving: an interpretive ethnography of the black Barbershop as cultural space. Qual Inquiry 2003;9:105-28.

30 Browne RC. Most Black women have a regular source of hair care-but not medical care. J Natl Med Assoc 2006;98:1652.

31 Versey HS. Centering perspectives on black women, hair politics, and physical activity. Am J Public Health 2014;104:810-5.

32 Hall RR, Francis S, Whitt-Glover M, et al. Hair care practices as a barrier to physical activity in African American women. JAMA Dermatol 2013;149:310-4.

33 Wilson TE, Fraser-White M, Feldman J, et al. Hair salon stylists as breast cancer prevention lay health advisors for African American and Afro-Caribbean women. J Health Care Poor Underserved 2008;19:216-26.

34 Sadler GR, Ko CM, Cohn JA, et al. Breast cancer knowledge, attitudes, and screening behaviors among African American women: the black cosmetologists promoting health program. BMC Public Health 2007;7:57.

35 Sadler GR, Ko CM, Wu P, et al. A cluster randomized controlled trial to increase breast cancer screening among African American women: the black cosmetologists promoting health program. J Natl Med Assoc 2011;103:735-45.

36 Sadler GR, Ko CM, Wu P, et al. Lessons learned from the black Cosmetologists promoting health program: a randomized controlled trial testing a diabetes education program. J Commun Healthc 2014;7:117-27.

37 Sadler GR, Thomas AG, Dhanjal SK, et al. Breast cancer screening adherence in African-American women. Cancer 1998;83:1836-9.

38 Sadler GR, Thomas AG, Gebrekristos B, et al. Black cosmetologists promoting health program: pilot study outcomes. J Cancer Educ 2000;15:33-7.

39 Smalls BL, Walker RJ, Bonilha HS, et al. Community interventions to improve glycemic control in African Americans with type 2 diabetes: a systemic review. Glob J Health Sci 2015;7:171-82.

40 Fitzgibbon ML, Tussing-Humphreys LM, Porter JS, et al. Weight loss and African-American women: a systematic review of the behavioural weight loss intervention literature. Obes Rev 2012;13:193-213.

41 Bland V, Sharma M. Physical activity interventions in African American women: a systematic review. Health Promot Perspect 2017;7:52-9

42 Barr-Anderson DJ, Adams-Wynn AW, DiSantis KI, et al. Familyfocused physical activity, diet and obesity interventions in AfricanAmerican girls: a systematic review. Obes Rev 2013;14:29-51. 
43 Lofton S, Julion WA, McNaughton DB, et al. A systematic review of literature on culturally adapted obesity prevention interventions for African American youth. J Sch Nurs 2016;32:32-46.

44 UyBico SJ, Pavel S, Gross CP. Recruiting vulnerable populations into research: a systematic review of recruitment interventions. J Gen Intern Med 2007;22:852-63.

45 Ndumele CD, Ableman G, Russell BE, et al. Publication of recruitment methods in focus group research of minority populations with chronic disease: a systematic review. J Health Care Poor Underserved 2011;22:5-23.

46 Moher D, Liberati A, Tetzlaff J, et al. Preferred reporting items for systematic reviews and meta-analyses: the PRISMA statement. PLoS Med 2009;6:e1000097.

47 Harris PA, Taylor R, Thielke R, et al. Research electronic data capture (REDCap)-A metadata-driven methodology and workflow process for providing translational research informatics support. $J$ Biomed Inform 2009;42:377-81.

48 Hoffmann TC, Oxman AD, loannidis JP, et al. Enhancing the usability of systematic reviews by improving the consideration and description of interventions. BMJ 2017;358:j2998.

49 Jackson N, Waters E, Guidelines for Systematic Reviews in Health Promotion and Public Health Taskforce. Criteria for the systematic review of health promotion and public health interventions. Health Promot Int 2005;20:367-74.

50 Armijo-Olivo S, Stiles CR, Hagen NA, et al. Assessment of study quality for systematic reviews: a comparison of the Cochrane collaboration risk of bias tool and the effective public health practice project quality assessment tool: methodological research. J Eval Clin Pract 2012;18:12-18. 